\section{Basale Meningitis mit Hirnstamminsult bei Morbus Boeck}

Der Befall des zentralen Nervensystems ist eine seltene Manifestation der Sarkoidose und aufgrund des uncharakteristischen klinischen Erscheinungsbildes schwierig zu diagnostizieren. Zusätzlich bestehen keine spezifischen nichtinvasiven Tests, so dass die Diagnose in der Annahme, dass es sich um eine Systemerkrankung mit Beteiligung des zentralen Nervensystems (ZNS) handelt, letztendlich durch das Erfüllen verschiedener Kriterien gestellt wird. Dazu gehören neben der neurologischen Symptomatik unter anderem der Kveim-Test, die Untersuchung des Liquors und der histologische Nachweis nichtverkäsender, epitheloidzelliger Granulome, der überlicherweise aus transbronchial gewonnenem Gewebematerial gelingt (Radziwill et al., Nervenarzt 1995; 66: 915). Einen besonders wichtigen Platz im diagnostischen Ablauf nimmt aufgrund ihrer hohen Sensitivität die kontrastmittelverstärkte Magnetresonanztomographie (MRT) ein (Pickuth et al., Radiologe 1999, 39: 889).

Wir berichten über eine junge Patientin, deren MRT die typischen Veränderungen beim Befall der basalen Meningen durch die Sarkoidose und als seltene Komplikation einen Hirnstamminsult dokumentierte.

\section{Fallbeschreibung}

Die 29-jährige Patientin litt seit bereits einem Jahr an rezidivierenden Kopfschmerzen, die sie als dauerhaften Druck empfand. Zusätzlich traten im letzten halben Jahr vor Hospitalisation 1 - 2-mal wöchentlich kurzdauernde Störungen der Sensibilität mit Parästhesien im linken Arm, Bein und in der linken Gesichtshälfte auf. Frühzeitig wurde eine neurologische Abklärung vorgenommen, wobei der klinische Status keine Ausfälle zeigte. Die nativ durchgeführte MRT zeigte keine intrakranielle Pathologie. Die Beschwerden wurden zu diesem Zeitpunkt als Migraine accompagnée interpretiert.

Im weiteren Verlauf kam es zu einer Zunahme der oben beschriebenen Symptome sowie zu einer Antriebsverminderung und einer Abnahme der Leis- tungsfähigkeit, wodurch der Verdacht auf eine zusätzliche depressive Symptomatik geäußert und die antidepressive Medikation begonnen wurde.

Am Tag der notfallmäßigen, stationären Aufnahme bemerkte die Patientin Kribbelparästhesien in der ganzen linken Körperhälfte, die sie in dieser Intensität noch nie erlebt hatte. In der klinischen Eintrittsuntersuchung fielen ein positives Babinski-Zeichen links, eine Pronation der linken oberen Extremität, ein Absinken der linken unteren Extremität und ein multidirektioneller Blickrichtungsnystagmus auf. Aufgrund dieser Befunde wurde der Verdacht auf einen Hirnstammprozess erhoben. Die MRT des Neurokraniums dokumentierte in der $\mathrm{T}_{2}$ - und der Diffusionswichtung eine umschriebene, im Territorium der rechtsseitigen, perforierenden, kurzen und langen zirkumferenten Äste der Art. cerebelli superior und Art. cerebri posterior (P2-Segment) lokalisierte Hyperintensität (Abb. 1, 2) im Hirnstamm, die einen deutlich erniedrigten Diffusionskoeffizienten aufwies (Abb.3). Nach der intravenösen Gabe von Kontrastmittel zeigte sich eine feinnoduläre leptomeningeale Anreicherung im Bereich der basalen Meningen und des Tentoriums mit einer markanten Verdickung des Hypophysenstieles (Abb.4), sowie deutlich weniger ausgeprägt über der Konvexität des supratentoriellen Hirnparenchyms. Im Hirnstamm fand sich keine Blut-HirnSchrankenstörung. Die Liquoruntersuchung ergab eine monozytäre Pleozytose von $49 \times 10^{6} / 1$ (Norm 1.1-1.9), eine Erhöhung des Gesamtproteins auf 1982 mg/l (Norm 180-480), ein erhöhtes Lactat von $2.8 \mathrm{mmol} / \mathrm{l}$ (Norm 1.1 1.9), ein erniedriger Glucosewert mit $1.1 \mathrm{mmol} / \mathrm{l}$ (Norm 2.2-4.2) und eine leicht erhöhte Aktivität des Angiotensin-converting-Enzyms von $7 \mathrm{U} / 1$ (Norm $<3$ ). Bis auf eine Hyponatriämie von $127 \mathrm{mmol} / \mathrm{l}$ (Norm 131-142) war die weitere Labordiagnostik des Liquors und des peripheren Blutes unauffällig, insbesondere gab es keine Hinweise auf die häufigsten zerebralen Infektionen (Herpes, Varizellen, FSME, Lues, Borelliose, HIV, Masern, Röteln, Tuberkulose). Aufgrund dieser Befunde wurde der Ver-

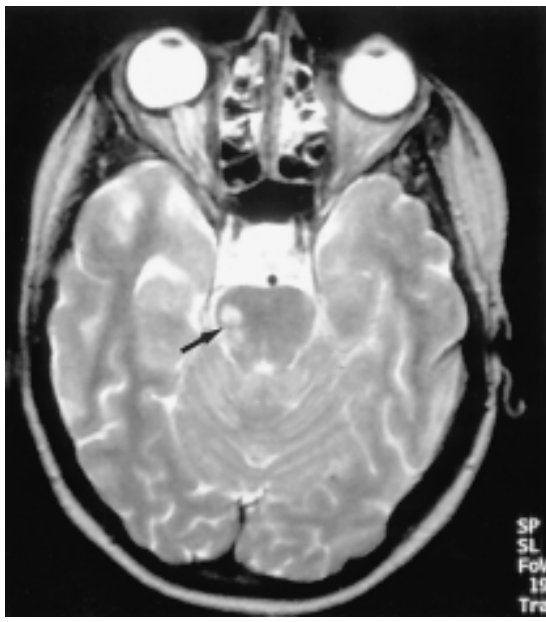

Abb.1 Hirnstamminfarkt: Die axiale $\mathrm{T}_{2}$-gewichtete (TSE-Technik, TR $3800 \mathrm{~ms}$, TE 90/ $2 \mathrm{~ms}$ ) Sequenz zeigt eine umschriebene, rechtsseitig lokalisierte Hirnstammläsion mit hoher Signalintensität (Pfeil).

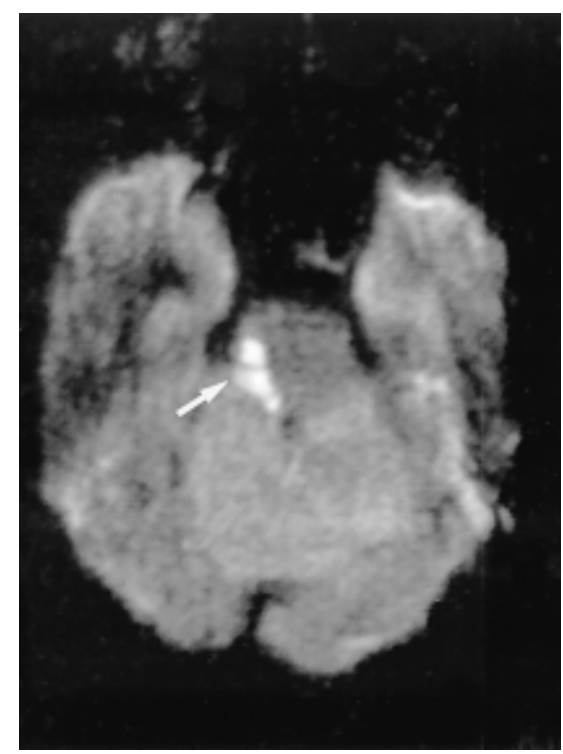

Abb. 2 Hirnstamminfarkt: Das axiale Diffusions-gewichtete Bild (EPI-SE-Technik, $\mathrm{b}_{\mathrm{o}}=1000$ ) zeigt eine hohe Signalintensität an gleicher Lokalisation wie das $\mathrm{T}_{2}$-gewichtete Bild (Pfeil).

dacht auf eine Neurosarkoidose mit einem Hirnstamminfarkt als Komplikation gestellt. Zur weiterführenden Diagnostik erfolgte die Computertomographie (CT) des Thorax: Es konnten pathologisch vergrößerte Lymphknoten im Mediastinum dargestellt werden; Hinweise für eine Mitbeteiligung des Lungenparenchyms bestanden nicht. Der Nachweis nichtverkäsender Granulome gelang anschließend bei der histologischen Aufarbeitung von transbronchial gewonnenem Biopsiematerial aus mediastinalen Lymphknoten. 


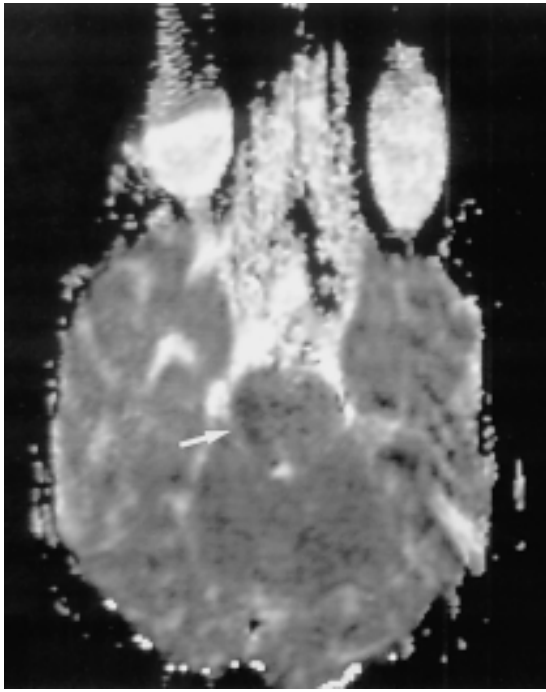

Abb. 3 Hirnstamminfarkt: Im axialen Diffusionskoeffizientenbild (ADC) weist die Läsion aus Abbildung 1 und 2 einen emiedrigten Diffusionskoeffizienten auf (Pfeil).

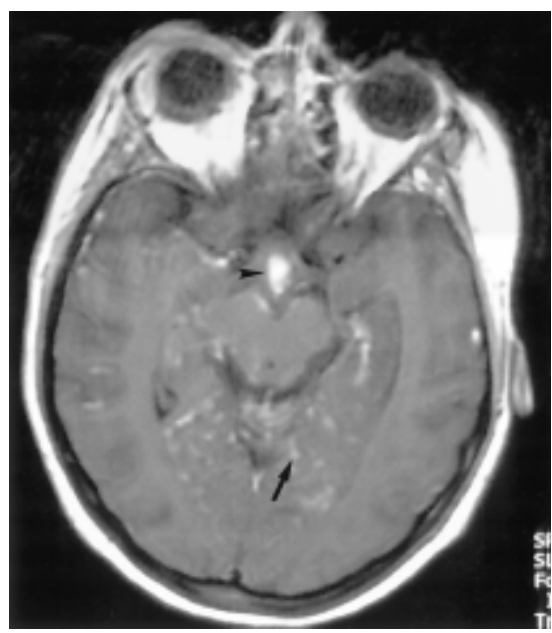

Abb. 4 Neurosarkoidose: In der axialen $\mathrm{T}_{1-}$ gewichteten MRT (SE-Technik, TR $600 \mathrm{~ms}$, TE 14/I ms) kommen nach intravenöser Kontrastmittelgabe feinnoduläre, meningeale Anreicherungen, die v.a. infratentoriell gelegen sind, zur Darstellung (Pfeil). Ferner zeigt sich eine Hyperintensität im Bereich des Hypophysenstiels (Pfeilspitze).

Die umgehend eingeleitete immunsuppressive Therapie mit initial hochdosierten Steroiden in Kombination mit Azathioprin führte zu einer schnellen Besserung der bei Eintritt bestandenen Symptomatik.

\section{Diskussion}

Ein Befall des ZNS, der zur klinischen Manifestation führt, wird bei der systemischen Sarkoidose in etwa $5 \%$ be- schrieben (Delaney et al., Ann Intern Med 1977, 42: 909). Dies bedeutet, dass bei einer Prävalenz der Sarkoidose von 20 50 Fälle/100 000 Individuen diejenige der Neurosarkoidose noch bei $1-2,5$ / 100000 liegt.

Die klinischen Symptome bei der Neurosarkoidose ergeben sich je nach befallenen Arealen. In über $50 \%$ gibt es mehr als eine Manifestation. Bevorzugt präsentiert sich die Neurosarkoidose durch Hirnnervenausfälle, wobei der N. facialis am häufigsten betroffen ist (Delaney et al., Ann Intern Med 1977, 42: 909). Eine weitere Prädilektionsstelle ist der Hypophysenstiel mit konsekutiver Funktionsstörung der Hypophysenhinterlappenhormone, insbesondere dem klinischen Bild eines Diabetes insipidus, der wie bei unserer Patientin subklinisch bleiben kann und erst aufgrund einer vorliegenden Hyponatriämie erkannt wird.

Unter dem Aspekt der uncharakteristischen Symptome und dem relativ seltenen Auftreten der Erkrankung bleibt die Neurosarkoidose ein diagnostisches Problem. Nebst dem klinischen Status wird der Liquor untersucht, der jedoch in 1/3 der Fälle keine Abnormalitäten aufweisen kann, der histologische Nachweis nichtverkäsender Granulome aus anderen Organen angestrebt und die kontrastmittelverstärkte MRT eingesetzt. Frühere und neuere Studien ergaben eine Sensitivität der MRT von $90 \%$, während die CT mit 77\% deutlich weniger geeignet ist zur Abklärung der Neurosarkoidose. Die Spezifität beider Untersuchungen ist indessen gering. Als häufigster MRT-Befund, mit rund 50\%, werden hyperintense Marklagerläsionen und periventrikuläre Läsionen in $\mathrm{T}_{2^{-}}$ gewichteten Bilder angegeben, die den Veränderungen der Multiplen Sklerose ähnlich sehen. Ebenfalls häufig (40$50 \%$ ) tritt nach Gadolinium-Gabe ein meningeales Enhancement von nodulärem Charakter im basalen und tentoriellen Bereich auf. Differenzialdiagnostisch sollte bei diesen Veränderungen an eine tuberkulöse Meningitis, an eine Meningeosis carcinomatosa oder an eine leukämische Infiltration gedacht werden. Seltener zeigen sich multiple, miliare supra- und infratentorielle Läsionen mit homogener Kontrastmittelanreicherung, die dem Bild intrazerebraler Metastasen gleichen. Des Weiteren werden mit geringer Häufigkeit $(5-10 \%)$ folgende Befunde als Komplikationen beschrieben: Hydrozephalus, intrabzw. extra-axiale solitäre Raumforderung, vergrößertes und hyperintenses Infundibulum (Pickuth et al., Radiologe 1999, 39: 889; Lexa et al., Am J Neuroradiol 1994, 15: 973). Selten sind als Komplikation der Sarkoidose zerebrale ischämische Läsionen beschrieben (Brown et al., Stroke 1989, 20: 400). Gemäß unseres Wissens wird in der Literatur bis anhin erst von einem Fall mit meningealem Enhancement und gleichzeitigem Infarkt im anliegenden Pons in der MRT berichtet, obwohl aus pathologischen Studien eine zerebrale Angiitis aufgrund der granulomatösen Entzündung der Adventitia seit langem bekannt ist (Lexa et al., Am J Neuroradiol 1994, 15: 973) und diese zu Hirnstamminfarkten führen kann. Als Pathomechanismus wird diskutiert, dass die primär leptomeningeale Entzündung entlang der Virchow-Robin-Räume ins Parenchym vordringt und dort entweder mechanisch oder entzündlich zu Verschlüssen der perforierenden Arterien führt. Die Diffusionsbildgebung erlaubt wie in unserem Fall durch die Darstellung des infarkttypischen, zytotoxischen Ödems eine Abgrenzung zu möglichen sarkoidosespezifischen intraparenchymatischen Läsionen.

Zusammenfassend lässt sich sagen, dass beim Vorliegen einer leptomeningealen Erkrankung in der MRT-Untersuchung mit Hypophysenstielbefall und feinnodulärem meningealen Enhancement, im Zusammenhang mit einem Diabetes insipidus und uncharakteristischen neurologischen Symptomen, eine Neurosarkoidose wahrscheinlich ist. Da in seltenen Fällen als Komplikation ischämische Veränderungen vorliegen können, sollten bei einem akut aufgetretenen Symptomenkomplex zusätzlich diffusionsgewichtete MRT-Sequenzen durchgeführt werden.

\section{Springende Punkte}

- seltene Manifestation der Sarkoidose

- uncharakteristische neurologische Symptome

- hohe Sensitivität der kontrastmittelverstärkten MRT

- zerebrale Ischämie als Komplikation möglich

U. Studler, Ph. Lyrer, A. Kaim, Basel 